\title{
The RNA strands of the plus and minus polarities of peach latent mosaic viroid fold into different structures
}

\author{
AUDREY DUBÉ, ${ }^{1}{ }^{\text {TILMAN BAUMSTARK, }}{ }^{2}$ MARTIN BISAILLON, ${ }^{1}$ and JEAN-PIERRE PERREAULT ${ }^{1}$ \\ ${ }^{1}$ RNA Group/Groupe ARN, Département de Biochimie, Faculté de Médecine et des Sciences de la Santé, Université de Sherbrooke, \\ Sherbrooke, Québec J1H 5N4, Canada \\ ${ }^{2}$ Department of Biological Sciences, University of the Sciences in Philadelphia, Philadelphia, Pennsylvania 19104, USA
}

\begin{abstract}
It is believed that peach latent mosaic viroid (PLMVd) strands of both the plus and minus polarities fold into similar secondary and tertiary structures. In order to verify this hypothesis, the behavior of both strands in three biophysical assays was examined. PLMVd transcripts of plus and minus polarity were found to exhibit distinct electrophoretic mobility properties under native conditions, to precipitate differently in the presence of lithium chloride, and to possess variable thermal denaturation profiles. Subsequently, the structure of PLMVd transcripts of minus polarity was elucidated by biochemical methods, thereby permitting comparison to the known structure of the plus polarity. Specifically, enzymatic probing, electrophoretic mobility shift assay, and ribonuclease $\mathrm{H}$ hydrolysis were performed in order to resolve the secondary structure of the minus polarity. The left domains of the strands of both polarities appear to be similar, while the right domain exhibited several differences even though they both adopted a branched structure. The pseudoknot P8 formed in the plus strand seemed not formed in the minus strands. The structural differences between the two polarities might have important implications in various steps of the PLMVd life cycle.
\end{abstract}

Keywords: viroid; RNA structure; circular RNA; probing; pseudoknot

\section{INTRODUCTION}

Viroids are small (250-400 nucleotides [nt]), circular, single-stranded RNAs which infect plants. Currently, $>30$ different viroids have been reported, and all belong to either the Pospiviroidae or the Avsunviroidae families. The former replicate in the nucleus, while the latter do so in the chloroplast. Viroid RNAs do not code for any protein; therefore, the integrity of their RNA structure is essential in order to ensure their complete life cycle. For example, it has been reported that some viroid RNA domains are critical for replication (Delgado et al. 2005; Zhong et al. 2006), transport (Zhong et al. 2008), or the induction of symptoms (Qi and Ding 2003), to name a few examples. Consequently, the determination of viroid secondary and tertiary structures appears to be critical to a better understanding of the various molecular mechanisms associated with the pathology of these infectious RNAs.

Reprint requests to: Jean-Pierre Perreault, RNA Group/Groupe ARN, Département de Biochimie, Faculté de Médecine et des Sciences de la Santé, Université de Sherbrooke, Sherbrooke, Québec J1H 5N4, Canada; e-mail: Jean-Pierre.Perreault@usherbrooke.ca; fax: (819) 564-5340.

Article published online ahead of print. Article and publication date are at http://www.rnajournal.org/cgi/doi/10.1261/rna.1826710.
Since viroids are relatively long RNA molecules, only a limited number of methods currently available can be used to evaluate their secondary structures. Computerassisted algorithms have frequently been used to predict the secondary structure of viroids (e.g., Bussière et al. 1996; Chaffai et al. 2007). However, this method possesses several limitations, including both the inability to accurately predict the pseudoknot structure and the fact that further validation of the proposed structure in solution is required. Biochemical methods have been used to deduce the structure of two viroids, specifically the potato spindle tuber viroid (PSTVd) and peach latent mosaic viroid (PLMVd). Plus polarity PSTVd transcripts, the classical representative of the Pospiviroidae family, adopt a rod-like secondary structure that was deduced from the first full sequence obtained for any viroid (Gross et al. 1978) and a host of physicochemical studies performed over the years (for review, see Owens 2007; Schmitz and Steger 2007). In addition, nuclease and chemical probing, divalent metal cationinduced cleavage, and UV crosslinking experiments largely confirmed these conclusions (Gast and Spieker 1996). However, a NMR study of a subdomain revealed significant differences in the structure resolution (Dingley et al. 2003). The branched structure adopted by the plus polarity 
transcripts of PLMVd, a member of the Avsunviroidae family, was deduced from analysis of both base-pair covariation and a combination of ribonuclease digestions and electrophoretic mobility shift assays (EMSA) with both RNA and DNA oligonucleotides (Bussière et al. 2000; Pelchat et al. 2000). Importantly, in both cases, the structures of transcripts of only one polarity were determined.

PLMVd replicates via a symmetric rolling-circle mechanism. Specifically, a host polymerase, which remains to be definitively identified, recognizes the circular monomer of one polarity and synthesizes linear multimeric conformers of the opposite polarity. The latter intermediates self-cleave releasing monomeric linear RNA strands that then selfligate, producing circular progeny. Because PLMVd possesses highly self-complementary sequences, which permit strands of opposite polarities to form similar stem-loop structures, and because the transcripts of both polarities share many biological features, for example, their replication mechanism resulting in symmetric amounts of both polarity RNAs, one might assume that they adopt nearidentical structures. Here, we report evidence challenging this intriguing hypothesis. To date, no work directed toward establishing the structure of the PLMVd transcripts of minus polarity has been performed. Several techniques that monitor physical properties demonstrated significant differences between the structures of PLMVd transcripts of plus and minus polarities. Subsequently, the secondary structure of the PLMVd strand of minus polarity was determined in solution, and was found to share several motifs with the plus polarity strand. However, several differences were also observed between the two strands.

\section{RESULTS AND DISCUSSION}

\section{Distinct biophysical behavior of PLMVd strands of both polarities}

In order to determine whether or not biophysical properties can be used to differentiate the PLMVd transcripts of plus and minus polarity, three biochemical analyses were performed. The first was electrophoresis of the RNA species under both denaturing and native conditions. Internally labeled linear plus and minus PLMVd transcripts were heat denatured and then fractionated on denaturing ( $8 \mathrm{M}$ urea) 5\% PAGE gels (Fig. 1A). Under these denaturing conditions the transcripts should migrate according to their molecular weight. As expected, since they are the same size (338 nt), both polarities migrated the same distance. Conversely, under native conditions, the secondary and tertiary structure of the RNA species should be preserved and should therefore influence their migration. Toward this end, internally labeled PLMVd transcripts of both polarities were heat denatured at $65^{\circ} \mathrm{C}$ and then slow cooled to $25^{\circ} \mathrm{C}$ in order to favor structural homogeneity. The RNA samples were then loaded on $5 \%$ polyacrylamide gels under native conditions (i.e., without urea). Surprisingly, a slight, but reproducible, difference in the position of the bands corresponding to the PLMVd plus and minus polarities was observed (Fig. 1B). Specifically, the migration of the minus polarity transcripts was always found to be slower
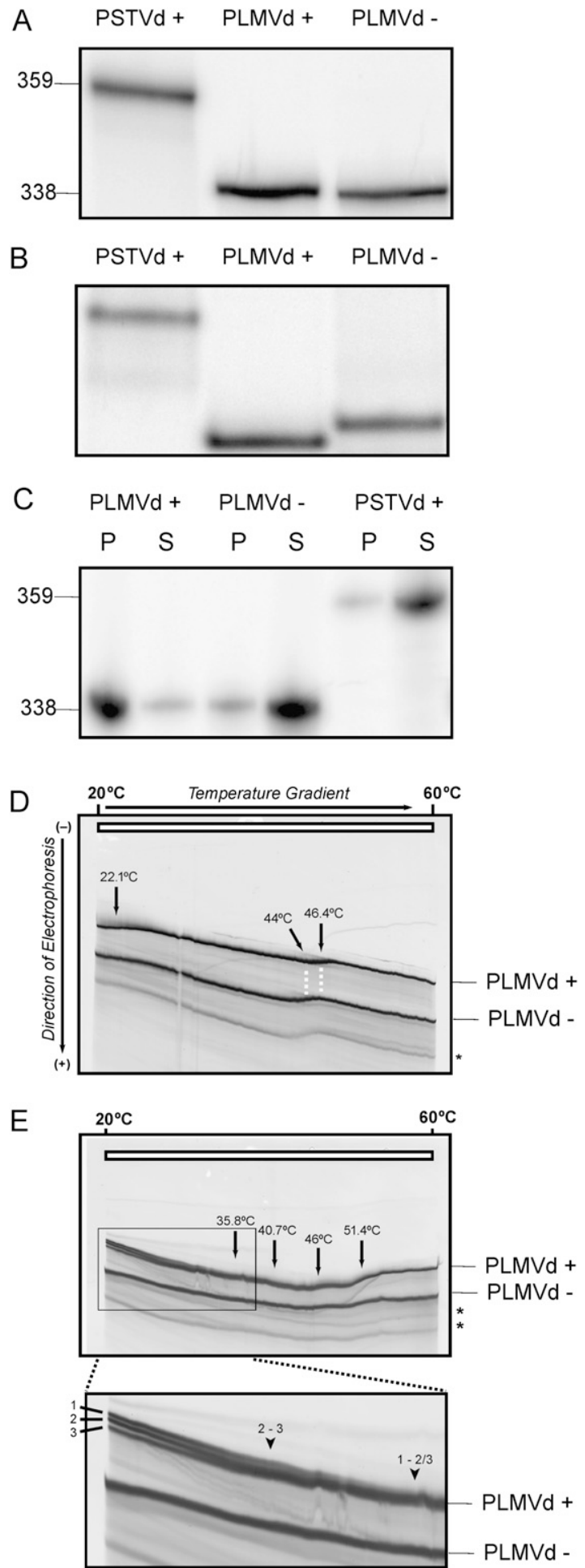

FIGURE 1. (Legend on next page) 
than that of the plus strands, suggesting that the structure of the latter is the more compact of the two. Even when the experiments were repeated under a variety of salt conditions (e.g., $50 \mu \mathrm{M} \mathrm{MgCl} 2$ or $150 \mathrm{mM} \mathrm{NaCl}$ ), equivalent results were always obtained (data not shown).

The second method used was lithium chloride $(\mathrm{LiCl})$ precipitation of the viroid RNA. One of the important physicochemical properties of viroids is their solubility in $2 \mathrm{M} \mathrm{LiCl}$ solution (Diener 2003). Generally, the viroids that fold into a rod-like structure, including PSTVd and the avocado sunblotch viroid (ASBVd), are highly soluble in LiCl. Conversely, viroids, such as the plus-stranded of PLMVd and chrysanthenum chlorotic mottle viroid (CChMVd), that adopt branched-like structures are insoluble in LiCl. Thus, we investigated whether or not the presence of $\mathrm{LiCl}$ caused the precipitation of PLMVd transcripts of both polarities. Internally radiolabeled RNA transcripts were heated and slowly cooled in either water or $50 \mathrm{mM}$ Tris- $\mathrm{HCl}$ ( $\mathrm{pH} 7.5$ ) $-10 \mathrm{mM} \mathrm{MgCl}$ solution prior to the addition of $\mathrm{LiCl}$ to a final concentration of $2 \mathrm{M}$. The samples were then incubated overnight at $-20^{\circ} \mathrm{C}$, centrifuged at $4^{\circ} \mathrm{C}$, the pellets stored, and the transcripts, if soluble, recovered from the supernatants by ethanol precipitation. All of the resulting pellets (i.e., $\mathrm{LiCl}$ and ethanol) were then dissolved in loading buffer and fractionated by electrophoresis through denaturing 5\% PAGE gels (Fig. 1C). Using an initial transcript concentration of $150 \mathrm{nM}$, $92 \%$ of the PLMVd strands of plus polarity precipitated in the presence of $\mathrm{LiCl}$, while only $6 \%$ of the PSTVd transcripts precipitated, in agreement with a previous report (Navarro and Flores 1997). Surprisingly, the PLMVd strands of minus polarity were $90 \%$ soluble in the presence of $2 \mathrm{M} \mathrm{LiCl}$ solution, with only $10 \%$ of these PLMVd minus transcripts precipitating under these conditions. Varying the transcript concentration $(10-300 \mathrm{nM})$ had no effect on the result. More importantly, these results showed that

FIGURE 1. Biophysical characterization of the PLMVd transcripts of both polarities. $(A, B)$ Autoradiograms of $5 \%$ PAGE gels run under denaturing $(A)$ and native $(B)$ conditions. PSTVd transcripts were used as molecular weight markers. The lengths, in nucleotides, of the transcripts are indicated on the right. $(C)$ Autoradiogram of a denaturing 5\% PAGE gel performed in conjunction with the lithium chloride precipitation experiment. $\mathrm{S}$ and $\mathrm{P}$ represent the supernatant and the pellet fractions, respectively. (D) TGGE analysis performed in $0.2 \times$ TBE buffer. Plus strand was applied 30 min later than the minus strand. The important transition temperatures are indicated by the arrows. (E) TGGE analysis of PLMVd transcripts performed in $0.2 \times$ TB buffer containing $10 \mu \mathrm{M}$ magnesium acetate. Plus strand was applied 30 min later than the minus strand. The sequential transitions are indicated by the arrows. The thin-lined box located at the bottom of the gel illustrates an area enlarged for better detail. The numbers on the left identify the three coexisting structures present at the lower temperature for plus polarity PLMVd transcripts. The arrowheads with the corresponding numbers designate where the transition curves of these coexisting structures merge due to conformational changes of the RNAs into a common intermediate. The asterisks represent the circular form of both polarities.
PLMVd strands of minus polarity were soluble in the presence of $2 \mathrm{M} \mathrm{LiCl}$, a known property of species (e.g., PSTVd) that fold into rod-like secondary structures.

Finally, PLMVd strands were analyzed by temperature gradient gel electrophoresis (TGGE), which permits not only both characterization and separation of structural transitions over a range of temperatures, but also the simultaneous detection of coexisting structures that would otherwise appear as an average conformation when either spectroscopic methods or chemical mapping approaches are used (for review, see Riesner and Steger 2005). Hence, PLMVd transcripts of both plus and minus polarities were first pretreated by heat denaturation followed by snap cooling in low salt buffer in order to eliminate bimolecular complexes and all conformations that might be trapped during either the transcription reaction or the gel extraction process. The transcripts of each polarity were initially analyzed in order to confirm their individual transition curves before applying them to the same temperature gradient gel. Analysis of the secondary structures detected using low ionic strength gels containing $0.2 \times$ TBE buffer indicated the presence of a single similar conformation for PLMVd transcripts of both the plus and minus polarities (Fig. 1D). Both polarities exhibited a lower temperature transition just above $20^{\circ} \mathrm{C}$, and a second major transition above $40^{\circ} \mathrm{C}$. Closer inspection, however, revealed that the change in mobility at $22^{\circ} \mathrm{C}$ was consistently more pronounced for the plus polarity, and that the midpoint of the main transition $\left(T_{\mathrm{m}}\right)$ for the transcripts of minus polarity was $2.4^{\circ} \mathrm{C}$ lower than for those of plus polarity. When a secondary treatment involving denaturing and slowly renaturing the transcripts in high salt buffer in order to enable formation of the lowest free energy structure, or for that matter, of any kind of structural equilibrium that might not form during a fast renaturation in low ionic strength buffer, was included following the low salt snap cooling, the same conformation was observed (data not shown). Thus, PLMVd transcripts of either polarity show little propensity to adopt alternative folds or metastable structures.

When PLMVd transcripts were subjected to TGGE in a buffer containing $10 \mu \mathrm{M}$ magnesium acetate to allow for formation of higher order structures including long-range or tertiary interactions that require divalent ions, transcripts of both polarities still appeared to adopt very similar conformations. Instead of one single high-temperature transition, they now denatured in a series of transitions between $35^{\circ} \mathrm{C}$ and $51^{\circ} \mathrm{C}$, indicating a differential stabilization of individual structural elements by the magnesium ions (Fig. 1E). This kind of sequential denaturation is consistent with the presence of a series of hairpins that form independently, each possessing its own distinct $T_{\mathrm{m}}$. A major difference between PLMVd transcripts of plus and minus polarities was detected at the lower temperature end of the gradient. More specifically, while PLMVd strands of minus polarity continued to appear as a single structure 
with a very weak transition above $20^{\circ} \mathrm{C}$, three distinct coexisting structures could be resolved for PLMVd transcript of plus polarity at the beginning of the temperature gradient (enlarged area in Fig. 1E). Two of the three transition curves merge into a single conformation, which in turn, merges with the structure of lowest mobility at a slightly higher temperature to form a single conformation, indicating a common overall structure with the potential to form local folding alternatives. Given that these coexisting structures were detected only in the presence of magnesium ions and at lower temperatures, these conformational alternatives could possibly involve hairpin-hairpin or other tertiary structural interactions. While the potential to form a pseudoknot, for example, has been described for PLMVd strands of plus polarity, it is noteworthy that transcripts of minus polarity do not show evidence of a similar type of interaction or alternative folds according to the TGGE analysis.

The results obtained from all three methods consistently indicated that PLMVd strands of minus polarity fold into a distinct structure different from that of their plus polarity counterparts. The minus polarity strands seemed to solely adopt a single, slightly less stable conformation. This result was unexpected given the initial hypothesis that strands of both polarities should adopt a globally similar structure. At this point it was not clear whether or not the variations of the physicochemical properties of the PLMVd strands resulted from subtle local structural variations, or from more significant and extended structural differences. In order to properly address this question it was necessary to determine the secondary structure of the PLMVd transcripts of minus polarity, as that of the strands of plus polarity had already been determined (Bussière et al. 2000).

\section{Determination of the secondary structure of PLMVd minus strands by RNase mapping}

Initially, ribonuclease (RNase) mapping was used in an attempt to determine the secondary structure of the PLMVd transcripts of minus polarity. Two distinct PLMVd species were probed. The first was synthesized from a dimeric PLMVd template from which the transcripts produced efficiently self-cleaved during runoff transcription, resulting in the release of PLMVd monomers with 5 '-ends corresponding to position 290 (i.e., the hammerhead self-cleavage site). The second species was synthesized from a PCR- amplified monomeric template in which the transcripts start at position 95. In the latter case, the three first nucleotides of the transcripts are consecutive guanosines, thus permitting efficient transcription. Mutation of the residues $\mathrm{U} 9$ to an $\mathrm{A}$ and $\mathrm{A} 330$ to a $\mathrm{U}$ were required to avoid hammerhead self-cleavage, resulting in the production of one-unit length transcripts (i.e., $338 \mathrm{nt}$ ). Analysis of the two different transcripts provided structural information for all positions of the PLMVd species even though the resolution of the electrophoresis is limited to $\sim 125-150$ nt. Experiments were performed using both $5^{\prime}\left[\gamma^{32} \mathrm{P}\right]$ ATP- and $3^{\prime}$ $\left[\alpha-{ }^{32} \mathrm{P}\right]-\mathrm{pCp}$ labeled transcripts. Prior to each experiment, the radiolabeled transcripts were heat denatured and slowly cooled in order to favor structural homogeneity. After this preliminary step, RNases were added and the reactions then incubated at $25^{\circ} \mathrm{C}$. Hydrolysis was performed using RNases A, T1, and TA, that cleave the $3^{\prime}$-phophodiester bonds of specific ribonucleotides ( $\mathrm{U}$ and $\mathrm{C}, \mathrm{G}$, and, $\mathrm{A}$, respectively) located in single-stranded regions, as well as with RNase V1 that cleaves double-stranded residues regardless of the base identity. In all cases an RNA carrier was omitted in order to avoid any interference with the PLMVd strands' structures.

A typical autoradiogram of a mapping gel is presented (Fig. 2) for the $5^{\prime}$-end labeled transcripts produced by hammerhead cleavage (i.e., starting at position 290). As an

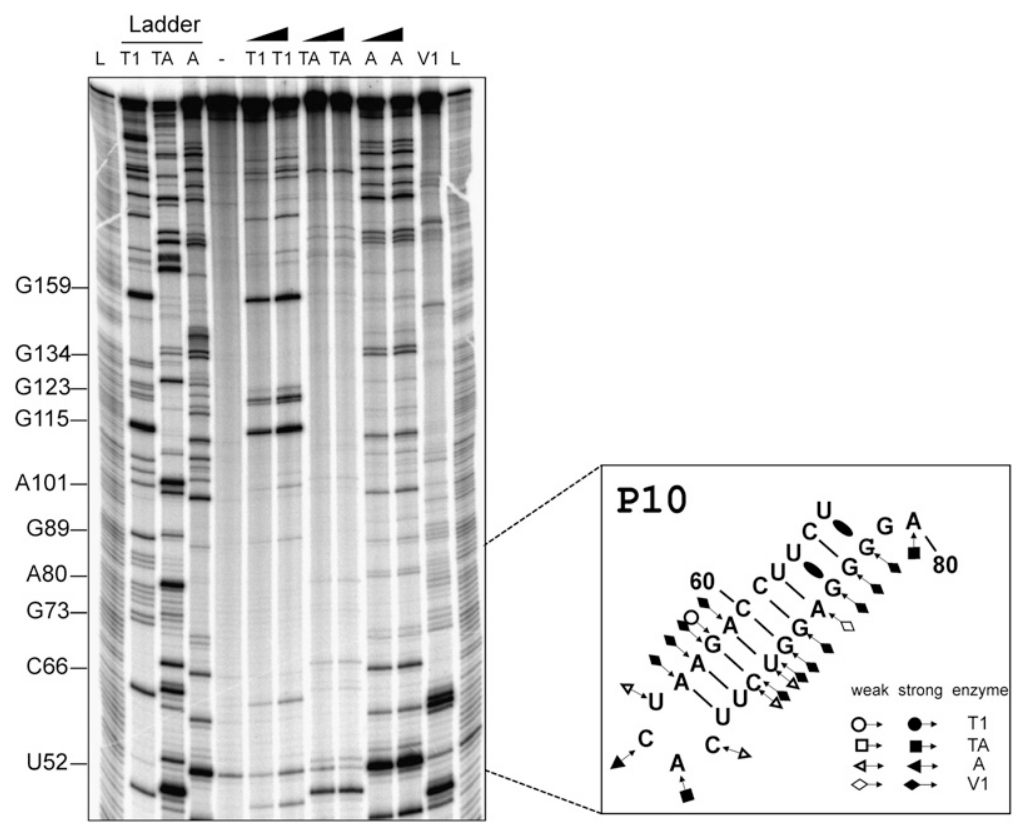

FIGURE 2. Typical autoradiogram of an $8 \%$ PAGE gel performed for the enzymatic probing of a 5'-end labeled PLMVd transcripts of minus polarity. The lanes labeled $\mathrm{L}$ on both sides are identical ladders obtained by alkaline hydrolysis of PLMVd. Lanes 2, 3, and 4 contain PLMVd transcripts hydrolyzed by RNase T1, RNase TA, and RNase A, respectively, under denaturing conditions in order to provide guanosine, adenosine, and cytosine/uridine ladders. Lane 5 is an unhydrolyzed RNA sample. The remaining lanes contained samples obtained from hydrolyzes performed using two dilutions of RNase T1 (lanes 6,7), RNase TA (lanes 8,9), RNase A (lanes 10,11), and one dilution of RNase V1 (lane 12). The inset on the right is a schematic compilation of the RNase probing of the P10 stem-loop. 
example the right inset in Figure 2 shows a schematic representation of the hydrolysis data for the region located at the bottom of the gel, which corresponds to the P10 stem-loop structure of the conformation. RNase V1 hydrolyzed after each nucleotide on both strands of the stem, although at different levels. Some residues of both strands were also hydrolyzed by specific singlestranded RNases, although at reduced levels (e.g., RNase T1 slightly cleaved after G62, and RNase A cleaved after both C71 and U72). Importantly, the four residues located in the loop were hydrolyzed only by the relevant specific single-stranded enzyme and not by RNase V1. Finally, the minus strand's P10 stem is longer than that of the plus polarity by three base pairs; consequently, the P9 stem-loop is shifted forward.

The mixtures produced with both PLMVd-derived transcripts were resolved using different electrophoretic conditions (both the acrylamide concentration and the migration times were varied). Moreover, all mapping experiments were repeated at least three times in order to provide a sufficiently high level of confidence in the mapping of each position. A compilation of the data for each nucleotide is presented in Supplemental Table 1. Incorporating all of the mapping data, a secondary structure can be proposed for PLMVd minus strand (Fig. 3A). Our results indicated that $212 \mathrm{nt}$ are involved in WatsonCrick base pairs, 10 in Wobble base pairs, and that 116 are located in singlestranded regions. Overall, this corresponds to $66 \%$ of the nucleotides being base-paired, and only $34 \%$ are found to be located in single-stranded regions. Of the 338 positions, 281 were placed at a high confidence level, while 57 remain ambiguous based simply on the RNase mapping hydrolysis results. For example, according to the proposed structure, the cytosine located in position 228 should have been cleaved by RNase A, which was not the case. This may simply be because the L3 loop was not accessible to the RNase A due to steric hindrance, or because this cytosine interacts with another residue.

In order to facilitate the comparison of the PLMVd strands of both polarities, we used the nomenclature of the PLMVd plus polarity strands' stem-loops with those from the strands of minus polarity (see Fig. 3A,B). Any stem-

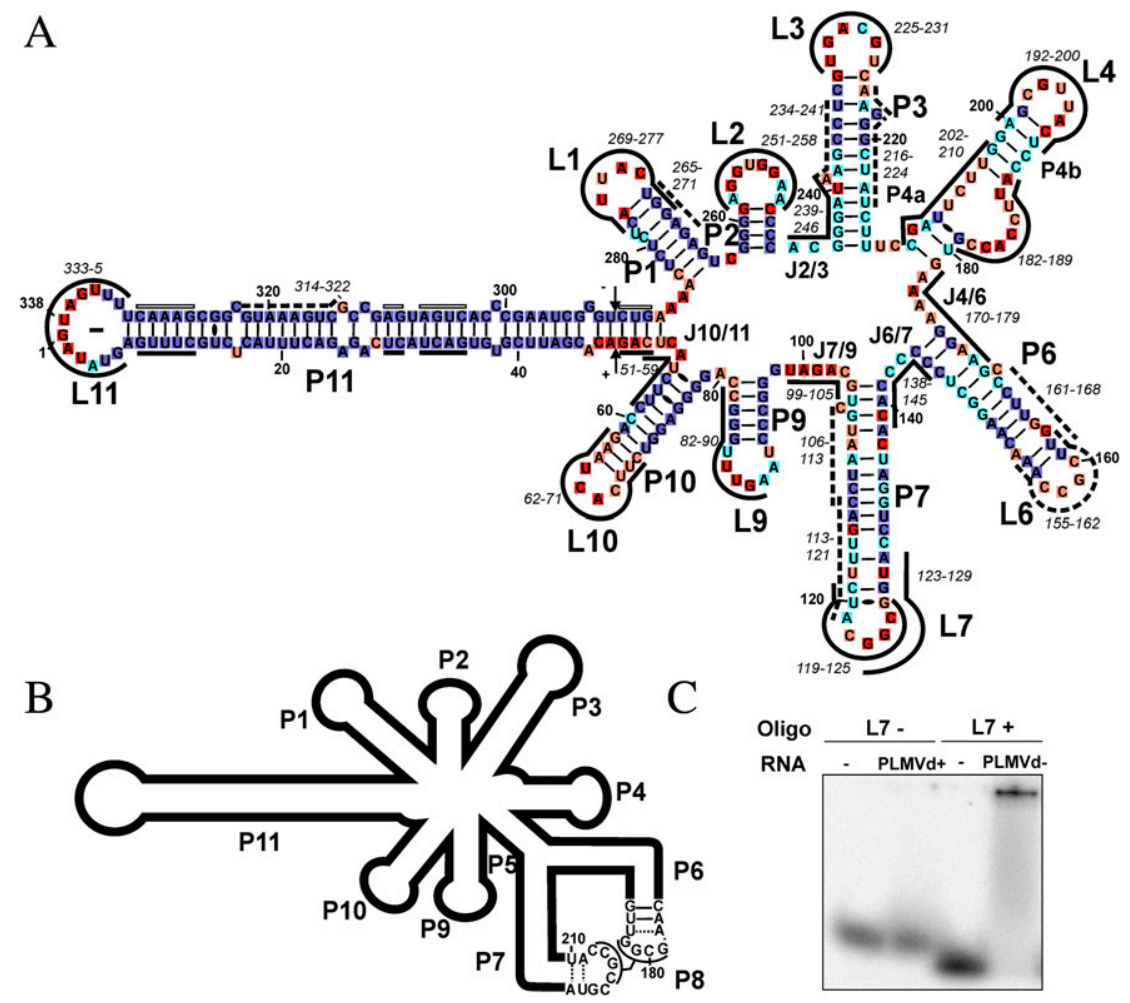

FIGURE 3. Proposed PLMVd secondary structures. (A) Compilation of the mapping results on the proposed secondary structure of the minus polarity PLMVd transcripts. The red boxes represent single-stranded nucleotides, while the blue ones designate the double-stranded dark blue boxes indicate unambiguous double-stranded nucleotides. The black and white structures, respectively. The oligonucleotides used in the EMSA and RNase $\mathrm{H}$ hydrolysis (loops and junctions) and unaccessible regions (stems), respectively. The nucleotide numbers of the viroid to which each oligonucleotide is complementary are indicated in italics above each oligonucleotide. All stems, loops, and junctions are identified. (B) Schematic represenstems are identified. The nucleotides involved in the formation of the P8 pseudoknot are also indicated. $(C)$ Autoradiogram of a EMSA performed in order to investigate the presence of the P8 pseudoknot. Lanes 1 and 2 are the experiments performed with the oligonucleotides complementary to sequence of the region of the L7 loop of plus polarity either in the absence or presence of the PLMVd transcripts of plus polarity. Lanes 3 and 4 are the experiments performed with the oligonucleotides complementary to sequence of the region of the L7 loop of minus polarity either in the absence or presence of the PLMVd transcripts of minus polarity.

loop structure missing in the PLMVd strands of minus polarity (compared with the plus polarity) were simply skipped. Overall, the structure is composed of nine stemloops, numbered from stem P1 to P11 (i.e., P1, P2, P3, P4, P6, P7, P9, P10, and P11). Briefly, the stem-loops P1-L1, $\mathrm{P} 2-\mathrm{L} 2$, and $\mathrm{P} 3-\mathrm{L} 3$ were virtually identical to their homologs in the plus polarity strand. Stem P4 is significantly longer in the PLMVd strands of minus polarity. In fact, the sequence of the P5 stem found in the plus strands is used in the minus strand to form the lower region of the longer P4 stem that is composed of 2 consecutive helices (P4a and $\mathrm{P} 4 \mathrm{~b})$. The sequence involved in the other strand of the lower portion of the $\mathrm{P} 4$ of the minus polarity strand forms a single-stranded junction between the P3 and P4 stems in 
the strands of plus polarity. Only a minimal junction of two nucleotides exists in the PLMVd minus polarity. The two regions of the $\mathrm{P} 4$ stem are connected by a relatively large internal loop composed of seven nonpaired nucleotides (positions 182 to 188) and 3 nucleotides (positions 204 to 206). The P6-L6 and P7-L7 stem-loops of the minus polarity strands are similar to those of the plus polarity strands. However, the P8 pseudoknot formed between the nucleotides composing the loop of the P6 and P7 stems in the strands of plus polarity appears to be missing in the minus polarity strands. The P9-L9 of the minus polarity strand is reminiscent of the one found in the plus polarity strand, but it is slightly shifted (by four positions), contains only $5 \mathrm{bp}$ instead of $6 \mathrm{bp}$, and is preceded by a singlestranded junction coming from the P7 stem. In the plus polarity strand the sequence forming that junction is part of the base-paired nucleotides that constitute part of the other strand of the P5 stem. The minus strand's P10 stem is $3 \mathrm{bp}$ longer in the proposed structure, and there is a shorter junction between the P10 and P11 stems compared with the plus strand (i.e., 2 nt versus $4 \mathrm{nt}$ ). Finally, the P11 stemloops are similar in both structures, with both corresponding to very long paired regions that include five helical domains (P11a to P11e) capped by a large loop.

In conclusion, the mapping experiments revealed a new and distinct secondary structure for the PLMVd transcripts of minus polarity. Overall, the resulting structure shares only the P11 stem with one obtained by computer assisted prediction (data not shown). The situation was similar for the previously reported structure of the PLMVd (+) strand (Bussière et al. 2000). The most important differences between the strands of plus and minus polarity are all located between stems $\mathrm{P} 4$ and P10, and, in terms of the minus polarity, consist of a reorganization of the sequences that form the P5 stem in the plus polarity strands and the omission of the P8 pseudoknot. With the exception of minor local differences, the structures of the stems from P10 to P2, which form the left domain, appear to fold into a stable structure similar to that found in the plus polarity transcript. Conversely, stems from $\mathrm{P} 3$ to $\mathrm{P} 9$ of the minus polarity, which form the right domain, appear to be less stable and more capable of adopting alternative structures compared with those of the plus strands. While no alternative structures were observed for the minus strands, TGGE did provide evidence for a decreased thermal transition of minus strand transcripts compared with the plus polarity. When the mapping experiments were performed using different salt conditions, no important differences were detected. In fact, only minor, local differences were observed and these did not influence the proposed secondary structure.

With the exception of the few isolated residues for which the determination of the single-stranded or doublestranded state was not certain, it seems that PLMVd strands of minus polarity do not adopt an alternative conformation under the conditions used here. This is in contrast to what is observed with the PLMVd strands of plus polarity for which part of the sequence of P11 stem has also been detected, to a small percentage at least, to be folded into one of the helices involved in the hammerhead secondary structure (Bussière et al. 2000). It is important to note that the experiments reported here were performed in the absence of any peach protein, a fact that may influence the folding of the viroid. For example, it has been shown that the presence of peach elongation factor eEF1A alters the viroid's structure near the junction between the P10 and P11 stems, adjacent to the self-cleavage site (Dubé et al. 2009). Likely, this is the binding site of the elongation factor, and its binding might contribute to the viroid's polymerization by the rolling circle replication.

\section{Support for the proposed secondary structure}

Concurrently with the RNase mapping experiments, the structure adopted by the transcripts of minus polarity was also studied by electrophoresis mobility shift assay, an approach based on the binding of complementary oligonucleotides. Twenty-three oligonucleotides that hybridize to different regions of PLMVd strands of minus polarity were synthesized. Some were designed to verify the accessibility of the proposed single-stranded regions of either the loops or the junctions between two stems, while others were designed to confirm the inaccessibility of proposed double-stranded regions (see Table 1). All oligonucleotides were relatively small ( 7 to $11 \mathrm{nt}$ in length) and offered a theoretical minimum Gibbs free energy $\left(\Delta G^{\circ}\right)$ at $37^{\circ} \mathrm{C}$ of $<-6 \mathrm{kcal} / \mathrm{mol}$ if the corresponding complementary sequence in the transcript is indeed in a single-stranded region, two criteria essential for the detection of the formation of an RNA-DNA heteroduplex. Initially, the transcripts produced by self-cleavage were heat denatured and slow cooled to room temperature in order to ensure structural homogeneity. The $5^{\prime}$-end labeled oligonucleotides were then added and the mixtures incubated at $25^{\circ} \mathrm{C}$ for 10 min prior to fractionation on native PAGE gels (see Supplemental Fig. 1 for a typical result). Seven oligonucleotides were synthesized in order to investigate the proposed double-stranded regions (Table 1, upper portion). In all cases no binding shifts were detected, supporting the formation of the corresponding stems. Eleven oligonucleotides were designed in order to confirm the proposed loop regions (Table 1, middle portion). With the exception of oligonucleotide 155-162 that targets loop L6, which may probably form dimers, binding shifts were observed in all cases, albeit at different levels. Finally, five oligonucleotides were designed in order to investigate the proposed junctions (Table 1, lower portion). In all cases, binding shifts were detected. For example, the junction J4/6, whose corresponding nucleotides in the plus polarity PLMVd strands are involved in the P5 stem, was confirmed as being accessible to the binding of oligonucleotide 170-179 based 
TABLE 1. Results of the EMSA and RNase $\mathrm{H}$ hydrolysis experiments

\begin{tabular}{|c|c|c|c|c|c|c|}
\hline DNA oligonucleotide & $\begin{array}{l}\text { Length } \\
\text { (nt) }\end{array}$ & $\begin{array}{c}\Delta G^{\circ} \text { at } 37^{\circ} \mathrm{C} \\
(\mathrm{kcal} / \mathrm{mol})\end{array}$ & Position & EMSA & $\begin{array}{l}\text { RNase H } \\
\text { cleavage }\end{array}$ & Region \\
\hline 5'-CATTTCAGC-3' & 9 & -8.6 & $314-322$ & - & - & P11b \\
\hline 5'-АССТСТС-3' & 7 & -9.0 & $265-271$ & - & - & $\mathrm{P} 1 \mathrm{~b}$ \\
\hline 5'-TATTCGGA-3' & 8 & -7.9 & $234-241$ & - & - & P3a \\
\hline 5'-TTCCCGATA-3' & 9 & -9.5 & $216-224$ & - & - & P3b \\
\hline 5'-GGAACCAA-3' & 8 & -7.9 & $161-168$ & - & - & P6b \\
\hline 5'-GGATTACG-3' & 8 & -7.2 & $106-113$ & - & - & P7 \\
\hline 5'-TAGAAACTG-3' & 9 & -7.9 & $113-121$ & - & - & P7 \\
\hline 5'-GTAATGACC-3' & 9 & -8.6 & $269-277$ & ++ & + & L1 \\
\hline 5'-ТCСАССТТ-3' & 8 & -8.3 & $251-258$ & + & ++ & L2 \\
\hline 5'-ACTGCAG-3' & 7 & -7.1 & $225-231$ & + & + & L3 \\
\hline 5'-GCTAAGAAC-3' & 9 & -8.2 & $202-210$ & + & - & $\mathrm{L} 4 \mathrm{a}$ \\
\hline 5'-TCGCAATGA-3' & 9 & -10.2 & $192-200$ & + & - & $\mathrm{L} 4$ \\
\hline 5'-TAAGGTGG-3' & 8 & -8.0 & $182-189$ & ++ & ++++ & $\mathrm{L} 4 \mathrm{~b}$ \\
\hline 5'-AAGCGGTT-3' & 8 & -8.9 & $155-162$ & - & + & L6 \\
\hline 5'-GCCGTAG-3' & 7 & -7.6 & $119-125$ & + & + & L7 \\
\hline 5'-TCAAACCCG-3' & 9 & -10.3 & $82-90$ & + & - & L9 \\
\hline 5'-GAAGTGATTC-3' & 10 & -9.0 & $62-71$ & + & - & L10 \\
\hline 5'-ATATCATCAAA-3' & 11 & -8.7 & $333-5$ & ++ & +++++ & L11 \\
\hline 5'-TGCССТАT-3' & 8 & -8.1 & $239-246$ & + & + & $J 2 / 3$ \\
\hline 5'-СТТTТССТТС-3' & 10 & -9.2 & $170-179$ & ++ & +++ & J4/6 \\
\hline 5'-GGGGGTGT-3' & 8 & -9.7 & $138-145$ & ++ & + & $J 6 / 7$ \\
\hline $5^{\prime}$-ACGTCTA-3' & 7 & -6.8 & 99-105 & + & - & $J 7 / 9$ \\
\hline 5'-GAAGATGAG-3' & 9 & -7.8 & $51-59$ & + & - & $J 10 / 11$ \\
\hline
\end{tabular}

For EMSA, the shifts were evaluated according to: $(-)$ indicates no shift, $(+)$ indicates that only a fraction of the oligonucleotides was shifted, and $(++)$ the totality of the oligonucleotides was shifted. For the RNase $\mathrm{H},(-)$ indicates no cleavage, $(+)$ a cleavage between 0.1 and $1 \%$, $(++)$ a cleavage between 1 and $5 \%,(+++)$ a cleavage between 5 and $10 \%,(++++)$ a cleavage between 10 and $30 \%$, and finally (+++++) a cleavage between $30 \%$ and $50 \%$.

on the EMSA assay. Similarly, both the J6/7 and J7/9 junctions that are not present in the proposed secondary structure for the plus polarity transcripts received physical support from the binding shift assays (see Fig. 3A and Table 1 for all of the EMSA data). Clearly, EMSA provides strong support for the proposed secondary structure of the PLMVd transcripts of minus polarity deduced from the RNase mapping experiments.

Complementary RNase $\mathrm{H}$ hydrolysis experiments on the RNA-DNA heteroduplexes were also performed (for a typical result, see Table 1; Supplemental Fig. 1). RNase H hydrolysis was detected for seven out of the 11 loops. The lack of hydrolysis in four cases (i.e., oligonucleotides 62-71 [loop L10], 82-90 [loop L9], 202-210 [loop 4a], and 192200 [loop L4]) might be caused by the inaccessibility of the enzyme to the heteroduplex, an additional limit of this approach as compared with EMSA. In contrast, RNase $\mathrm{H}$ hydrolysis of the heteroduplexes formed with the oligonucleotide 155-162 that is complementary to the L6 loop was observed, albeit at a reduced level, even though no electrophoretic mobility shift had been detected. In this experiment the observed cleavage product's size was as expected for binding to the L6 loop, confirming at least its transient existence. The presence of the loops L4, L6, and L10 received additional physical supports by modification of the single-stranded cytosine residues by dimethyl sulfate (DMS) probing coupled to subsequent cleavage with hydrazine and aniline (data not shown). Only the loop 9, which did not include a cytosine residue, cannot be definitively proved. Finally, three of the five oligonucleotides complementary to the junctions bound their corresponding domains, based on RNase $\mathrm{H}$ hydrolysis results (see Table 1). This confirms the presence of all proposed junctions based on RNase mapping.

\section{Absence of pseudoknot structure}

According to the data presented above, there is no evidence suggesting the presence of a pseudoknot in the PLMVd transcripts of minus polarity. This is in contrast to what is observed with the transcripts of plus polarity where the P8 pseudoknot is formed via base pairing between nucleotides from the L6 and L7 loops (Fig. 3B; Bussière et al. 2000). The eight nucleotides involved in this pseudoknot are perfectly conserved in all PLMVd sequence variants reported to date (i.e., ${ }_{179} \mathrm{GCGG}_{182}$ and ${ }_{212} \mathrm{CCGC}_{215}$, respectively). This suggests that this pseudoknot is critical in some aspect of the viroid's biology, although covariation for base-paired nucleotides would have been even more definitive proof of this conclusion. Regardless, this conclusion is supported by 
the detection of an equivalent pseudoknot in the secondary structure of CChMVd, another member of the Avsunviroidea family (Bussière et al. 2000). In order to further confirm the presence of this pseudoknot in the secondary structure of PLMVd plus polarity strands, an oligonucleotide complementary to the L7 loop was synthesized (5'-CGGC GGT-3') and tested in an EMSA experiment. Either no binding shift was detected (Fig. 3C, lane 2), or only a tiny amount of heteroduplex was observed, suggesting that the L7 loop is located within a double-stranded structure. When an oligonucleotide complementary to the corresponding sequence of the PLMVd minus transcript (5'-TACCGCC-3') was tested with full-length PLMVd minus polarity strands, a significant binding shift was detected (Fig. 3C, lane 4). This result indicated that the sequence of the minus polarity that corresponds to the L7 loop of the plus strand was accessible to the oligonucleotide. Together, the RNase probing data and the EMSA experiments with two oligonucleotides designed to investigate the L6 and L7 loops clearly show that there is no equivalent to the P8 pseudoknot of the plus strands in the PLMVd minus transcripts. At the limit, if there is a P8 pseudoknot formed by the minus strands PLMVd, this is only a small proportion of the RNA strands that include it.

The analysis of the proposed secondary structures adopted by the transcripts of the two polarities revealed that the environment of the nucleotides potentially involved in the formation of the P8 pseudoknot differed depending on the polarity of the strand. In the plus polarity the $8 \mathrm{nt}$ involved in forming the pseudoknot would be single stranded if this helix is not formed (Fig. 3B), whereas in the case of the minus polarity the $4 \mathrm{nt}$ of the L6 loop are single stranded, two are found in the L7 loop and two other are base paired (Fig. 3A). This arrangement leaves either only $2 \mathrm{nt}$ available for potential base pairing in the pseudoknot, or creates a competition between potential base-pairing partners for the nucleotides that would form a pseudoknot. Both of these scenarios are not favorable for the formation of a stable pseudoknot, and might explain why it seems to not be formed in the minus polarity transcripts. Interestingly, the equivalent pseudoknot was also found to be absent in the minus polarity CChMVd strands (Gago et al. 2005).

In order to investigate whether or not the P8 pseudoknot of the plus polarity transcripts is important in the viroid's life cycle, peach trees were inoculated using both wild-type and P8 mutant (i.e., variant 151.1 and ${ }_{212} \mathrm{CCGC}_{215} \rightarrow$ ${ }_{212} \mathrm{AAAA}_{215}$ mutations) unit-length PLMVd transcripts. A year after inoculation, PLMVd were detected in most of the trees inoculated with the wild-type sequence (A Dubé, O Parisi, J-P Perreault, and J Haissam, unpubl.). Conversely, no evidence of PLMVd was detected in the trees infected with the P8 pseudoknot mutant. Clearly, the presence of the P8 pseudoknot in the plus polarity is essential for the PLMVd life cycle, although its precise contribution remains to be determined.

\section{Concluding remarks}

This study presented biophysical evidence that permits us to establish a difference between the two polarities of PLMVd strands and proposes a model for the secondary structure of the minus polarity transcript. Cleary, both polarities fold into structures that exhibit both similarities and differences. For PSTVd, which replicates via an asymmetric rolling circle mechanism, metastable elements specific for plus and minus strands, respectively, drive folding into different structures that are critical for plus strand synthesis from minus strand replication intermediates $(\mathrm{Qu}$ et al. 1993; Repsilber et al. 1999; Schroeder and Riesner 2002) and for processing of multimeric plus strands into circular progeny (Baumstark et al. 1997; Schrader et al. 2003). For PLMVd, it is tempting to speculate that a high level of similarity was to be expected since this viroid replicates in a symmetrical manner and the intermediates of both polarities accumulate in roughly the same proportions in infected cells. However, the structural differences observed are more likely largely responsible for the distinct biophysical characteristics of the two polarities, and could also play a role in other aspects of the PLMVd life cycle. For example, one polarity of PLMVd could act as either an antisense RNA, or as a trans-acting ribozyme. In this line of thinking the minus polarity transcript, which adopts a less compact structure, is the more likely of the two to play such a role. In addition, the differences between the two polarities may help explain the differences observed when studying PLMVd strands of both polarities, differences such as the observation that the efficiencies of both self-cleavage and self-ligation in vitro varied between the two polarities. More specifically, the PLMVd strands of plus polarity self-cleaved in vitro at a level of $60 \%-70 \%$, while their counterparts of minus polarity did so only at $50 \%-55 \%$ under the same reaction conditions (Beaudry et al. 1995). Conversely, the minus strands self-ligated more efficiently than the plus transcripts (i.e., 5\%-8\% as compared with 1\%-2\%, respectively) (F Bolduc and J-P Perreault, unpubl.). In both cases these results might be explainable if the P11-containing secondary structure with the lower free energy than the alternative conformation, including the hammerhead motif, is favored in the minus polarity PLMVd transcripts (compared with what is occurring with the plus polarity). Specifically, the level of self-cleavage is reduced if the formation of the long P11 stem is favored because its formation reduces the time that the RNA strand has in which to adopt the hammerhead secondary structure (Beaudry et al. 1995). Moreover, the greater stability of the P11 stem would be expected to favor higher levels of self-ligation because it is this structure that supports the nonenzymatic reaction (Lafontaine et al. 1995). Interestingly, the minus polarity PLMVd strands did not permit the detection of alternative structures for the P11 stem domain, while those of plus polarity not only primarily 
folded into the long rod-like domain, but also permitted the formation of the hammerhead motifs (Bussière et al. 2000). On the other hand, the plus polarity PLMVd species is the more infectious of the two as shown by PLMVd inoculations into peach trees (Ambros et al. 1998). As previously proposed for the plus polarity PLMVd transcripts (Pelchat et al. 2000), those of minus polarity also appear to be composed of two domains: (1) The left domain that includes the P1-L1, P10-L10, and P11-L11 stem-loops formed by the hammerhead sequences and all of the features known to participate in the replication; and, (2) the right domain that includes all other stem-loops and to which no function has yet been attributed. Interestingly, all significant structural differences between the strands of both polarities were located in the right domain. It is important to understand that in the case of PLMVd, the unitlength transcripts of both polarities accumulated in large amounts in infected cells. To date, there is no evidence to suggest a more important role for one polarity compared with the other. Clearly, determination of the secondary structure of both strands constitutes an important step in elucidating their roles in the biology of this viroid.

\section{MATERIALS AND METHODS}

\section{RNA synthesis}

The synthesis and purification of both plus and minus PLMVd transcripts were performed as described previously (Bussière et al. 2000). Briefly, in vitro transcriptions were performed using digested recombinant plasmid pPD1, which contains two tandemly repeated PLMVd sequences cloned into the PstI restriction site of pBluescript II KS, as a template (Beaudry et al. 1995). In this construct the insert is flanked by the T3 and T7 promoters for the production of the plus and minus polarity transcripts, respectively. The transcription reactions were performed for $3 \mathrm{~h}$ at $37^{\circ} \mathrm{C}$ in a final volume of $100 \mu \mathrm{L}$ containing $80 \mathrm{mM}$ HEPES-KOH $(\mathrm{pH}$ 7.5), $24 \mathrm{mM} \mathrm{MgCl}_{2}, 2 \mathrm{mM}$ spermidine, $40 \mathrm{mM}$ DTT, $5 \mathrm{mM}$ of each NTP, $0.004 \mathrm{U} / \mu \mathrm{L}$ pyrophosphatase (Roche Diagnostics), 37 U RNA Guard (Amersham Biosciences), and $10 \mu \mathrm{g}$ of purified T7 or T3 RNA polymerase. For random internal labeling, $30 \mu \mathrm{Ci}$ of $\left[\alpha-{ }^{32} \mathrm{P}\right]$ UTP $(3000 \mathrm{Ci} / \mathrm{mmol}$; New England Nuclear) were added to the transcription reactions. During transcription, RNA molecules of both polarities self-cleaved, yielding 338-nt linear monomeric species. The transcriptions were stopped by the addition of RNase-free RQ1 DNase (Promega), followed by incubation for $30 \mathrm{~min}$ at $37^{\circ} \mathrm{C}$. One volume of stop buffer $(0.03 \%$ [wt/vol] each of bromophenol blue and xylene cyanol, $10 \mathrm{mM}$ EDTA [pH 7.5] and $97.5 \%$ [vol/vol] deionized formamide) was then added and the resulting mixtures denatured for $2 \mathrm{~min}$ at $65^{\circ} \mathrm{C}$ prior to being fractionated by denaturing ( $8 \mathrm{M}$ urea) $5 \%$ polyacrylamide gel electrophoresis (PAGE, 19:1 ratio of acrylamide/bisacrylamide) using $45 \mathrm{mM}$ Tris-borate ( $\mathrm{pH} 7.5)$ and $1 \mathrm{mM}$ EDTA as buffer $(1 \times$ TBE buffer). Nonradioactive transcripts were detected by UV shadowing, while radioactive ones were detected by autoradiography. The bands corresponding to the $338 \mathrm{nt}$ full-length fragments of both polarities were excised, the RNA eluted overnight in elution buffer (500 $\mathrm{mM} \mathrm{NH}_{4} \mathrm{OAc}, 1 \mathrm{mM}$ EDTA, and 0.1\% SDS), ethanol precipitated, purified on Sephadex G-50 spin columns (Amersham) and lyophilized. After dissolving in ultrapure water, the RNA concentrations were determined either by absorbance spectrophotometry at $260 \mathrm{~nm}$ or by Cerenkov counting, and the samples were then stored dry at $-20^{\circ} \mathrm{C}$.

In order to produce PLMVd transcripts starting at position 95, a PCR amplification using the oligonucleotides sense 5' -TAATAC GACTCACTATAGGGTAGACGTCGTAATCCAGTTTC-3' and antisense $5^{\prime}$-GGGATTCAAACCCGGTCCCCTCC-3' as primers was initially performed using Pwo DNA polymerase (Roche Diagnostic) and $0.01 \mu \mathrm{g}$ of pPD1 BamHI digested plasmid. After amplification, the proteins were removed by phenol-chloroform extraction and the cDNA precipitated with ethanol. The resulting pellets were dissolved in water and used directly in the transcription reactions.

One monomer of PSTVd isolate KF-440-2 (Schnoelzer et al. 1985) was cloned in pCDNA3 (Invitrogen) at the HindIII and EcoRI sites with two oligonucleotides: Sense oligonucleotide 1-PSTVdplus 5'-GACTCACTATTAGGAAGCTTCGGAACTAAAC-3', and antisense 359-PSTVdplus, 5' -TGAGCTGTATTAGAATTCAGGAACCA AC- $3^{\prime}$. This plasmid was linearized by EcoRI digestion and transcriptions were performed as described above with T7 RNA polymerase and $5 \mu \mathrm{g}$ of digested plasmid.

\section{5 ' - and $3^{\prime}$-end labeling}

Purified 338 nt PLMVd transcripts of minus polarity starting at either position 95 or position 290 (i.e., at the hammerhead cleavage site) were labeled as previously described (Bussière et al. 2000). Briefly, transcripts (25 pmol) were dephosphorylated in a final volume of $10 \mu \mathrm{L}$ using $10 \mathrm{U}$ of Antarctic phosphatase according to the manufacturer's procedure (New England Biolabs). The reactions were stopped by heating for $8 \mathrm{~min}$ at $65^{\circ} \mathrm{C}$. Subsequently, dephosphorylated transcripts $(5 \mathrm{pmol})$ were $5^{\prime}$-end labeled in the presence of $3.2 \mathrm{pmol}\left[\gamma_{-}{ }^{32} \mathrm{P}\right]$-ATP $(6000 \mathrm{Ci} / \mathrm{mmol}$, New England Nuclear) and $3 \mathrm{U}$ of T4 polynucleotide kinase according to the manufacturer's procedure (USB). The reactions were performed at $37^{\circ} \mathrm{C}$ for $60 \mathrm{~min}$. For $3^{\prime}$-end labeling, purified PLMVd transcripts $(20 \mathrm{pmol})$ were incubated with $10 \%$ DMSO, $40 \mu \mathrm{Ci}$ of $\left[\alpha-{ }^{32} \mathrm{P}\right] \mathrm{pCp}$ (3000 mCi/mmol; New England Nuclear), and $40 \mathrm{U}$ of purified T4 RNA ligase in a final volume of $10 \mu \mathrm{L}$ containing $50 \mathrm{mM}$ Tris- $\mathrm{HCl}$ ( $\mathrm{pH} 7.8), 10 \mathrm{mM} \mathrm{MgCl}_{2}, 1 \mathrm{mM}$ ATP, and $10 \mathrm{mM}$ DTT. After $60 \mathrm{~min}$ of incubation at $37^{\circ} \mathrm{C}$, the reactions were stopped by the addition of one volume formamide dye buffer and the mixtures then fractionated through denaturing 5\% PAGE gels. For $3^{\prime}$-end labeling of the transcripts resulting from the hammerhead self-cleavage, a T4 polynucleotide kinase (PNK) incubation was performed as described above, with the exception that the ATP was omitted in order to favor the reverse reaction and thus remove the $2^{\prime}-3^{\prime}$-phosphocyclic group (Amitsur et al. 1987). The PNK was then removed by two successive phenol-chloroform extractions, and the transcripts recovered by ethanol precipitation prior to performing the ligation reactions. After autoradiography, the bands containing the appropriate $5^{\prime}$ - and $3^{\prime}$-end-labeled transcripts were excised and the RNA recovered as described above.

\section{Migration of PLMVd transcripts in denaturing and native PAGE gels}

Internally radiolabeled transcripts $(\sim 10,000 \mathrm{cpm})$ were dissolved in $10 \mu \mathrm{L}$ of water and added to an equivalent volume of stop 
buffer. These mixtures were denatured for $2 \mathrm{~min}$ at $65^{\circ} \mathrm{C}$ prior to being fractionated by denaturing ( $8 \mathrm{M}$ urea) 5\% PAGE electrophoresis (19:1 ratio of acrylamide/bisacrylamide) using $1 \times \mathrm{TBE}$ buffer. The gels were run at $30 \mathrm{~W}$ for $2 \mathrm{~h}$. In the case of the native gels, TB buffer ( $45 \mathrm{mM}$ Tris-borate at $\mathrm{pH} 7.5)$ in the presence of either $150 \mathrm{mM} \mathrm{NaCl}$ or $10 \mu \mathrm{M}$ magnesium acetate was used. The radiolabeled transcripts were heated for $5 \mathrm{~min}$ at $65^{\circ} \mathrm{C}$ and were then slowly cooled to $25^{\circ} \mathrm{C}$. One volume of native loading buffer $(0.03 \%$ [wt $/ \mathrm{vol}]$ each of bromophenol blue and xylene cyanol in $50 \%$ glycerol in $1 \times \mathrm{TB}$ solution) were added prior to loading on $5 \%$ native polyacrylamide gels (29:1 ratio of acrylamide/bisacrylamide, no urea). The gels were run at $120 \mathrm{~V}$ for $10 \mathrm{~h}$ and then autoradiographed.

\section{Lithium chloride precipitation}

A mixture of radioactive $(\sim 10,000 \mathrm{cpm})$ and nonradioactive transcripts $(10,75,150$, and $300 \mathrm{nM})$ were heated at $65^{\circ} \mathrm{C}$ and then slowly cooled to $25^{\circ} \mathrm{C}$ in the presence of either water or $50 \mathrm{mM}$ Tris- $\mathrm{HCl}$ at $\mathrm{pH} 7.5 / 10 \mathrm{mM} \mathrm{MgCl}_{2}$ buffer. $\mathrm{LiCl}$ was then added to a final concentration of $2 \mathrm{M}$ and the mixtures completed to a final volume of $100 \mu \mathrm{L}$ with water. The precipitation reactions were then incubated overnight at $-20^{\circ} \mathrm{C}$ prior to centrifuging at $17,000 \mathrm{~g}$ for $30 \mathrm{~min}$. The supernatants were removed and the RNA ethanol precipitated from them. All pellets were ethanol washed, dried, and then dissolved in $10 \mu \mathrm{L}$ water and $10 \mu \mathrm{L}$ of denaturing loading buffer. The samples were fractionated on denaturing 5\% PAGE gels. After electrophoresis the gels were exposed to phosphor screens and then revealed using a Storm scanner (Molecular Dynamics). The ratios of the precipitated RNAs were then calculated using the Image Quant software.

\section{Temperature gradient gel electrophoresis}

Gel-purified full-length transcripts of PLMVd strands of both plus and minus polarity were denatured in TE buffer $(10 \mathrm{mM}$ Tris- $\mathrm{HCl}$ at $\mathrm{pH} 8.0$ and $1 \mathrm{mM}$ EDTA) and rapidly cooled in an ethanol/dry ice bath as described previously for PSTVd (Baumstark and Riesner 1995). In order to permit the formation of structures that are potentially disfavored by this treatment driven by fast kinetics, some transcripts were subsequently denatured and slowly renatured in a high salt buffer $(500 \mathrm{mM} \mathrm{NaCl}, 1 \mathrm{mM}$ cacodylic acid, $0.1 \mathrm{mM}$ EDTA at $\mathrm{pH} 7.0$ ) and then dialyzed against TE buffer on Millipore swim filters for $2-3 \mathrm{~h}$ at $4^{\circ} \mathrm{C}$. The resulting RNA structures were analyzed by temperature gradient gel electrophoresis (Rosenbaum and Riesner 1987) by loading $500 \mathrm{ng}$ of PLMVd transcripts of minus polarity to gels containing $5 \%$ acrylamide $/ 0.17 \%$ bisacrylamide in either $0.2 \times \mathrm{TBE}$ or $0.2 \times \mathrm{TBM}$ (TBE with $10 \mu \mathrm{M} \mathrm{MgOAc}$ instead of EDTA) buffer and then performing electrophoresis under native conditions for $30 \mathrm{~min}$ at $400 \mathrm{~V}$. Subsequently, $500 \mathrm{ng}$ of PLMVd strands of plus polarity were applied under the same conditions and allowed to enter the gel for $10 \mathrm{~min}$. Following the $10 \mathrm{~min}$ interval that is required in order to establish a temperature gradient from $20^{\circ} \mathrm{C}-60^{\circ} \mathrm{C}$, the electrophoresis was continued at $400 \mathrm{~V}$ for $60-80 \mathrm{~min}$. RNA structural transitions were visualized by silver staining (Schumacher et al. 1986).

\section{Enzymatic probing of PLMVd strands}

In all cases of enzymatic probing, trace amounts of slowly cooled either $5^{\prime}$ - or $3^{\prime}$-end-labeled $338 \mathrm{nt} \operatorname{PLMVd}(<1 \mathrm{nM}, \sim 10,000$ cpm) were dissolved in $2 \mu \mathrm{L}$ of water. The resulting RNA mixtures were then diluted to a final volume of $10 \mu \mathrm{L}$ that contained final concentrations of $10 \mathrm{mM}$ Tris- $\mathrm{HCl}(\mathrm{pH}$ 7.5), $0.5 \mathrm{mM}$ DTT, and $50 \mathrm{mM} \mathrm{NH}_{4} \mathrm{Cl}$. Solely in the case of the reactions performed in the presence of RNase $\mathrm{V} 1$, the solution also contained a final concentration of $5 \mathrm{mM} \mathrm{MgCl}_{2}$. The mixtures were incubated for $1 \mathrm{~min}$ at $25^{\circ} \mathrm{C}$ in the presence of either 0.16 or $0.25 \mathrm{U}$ of RNase T1 (Roche), 0.25 or $0.5 \mathrm{U}$ of RNase TA (Jena Bioscience), 0.1 or $1 \mathrm{pg} / \mu \mathrm{L}$ RNase A (USB), or $0.02 \mathrm{U}$ of RNase V1 (Pierce Molecular Biology), and were quenched, after the incubation, by the addition of $10 \mu \mathrm{L}$ of $50 \%$ formamide and $10 \mathrm{mM}$ EDTA solution. In order to prepare different RNA ladders, transcripts were diluted in a final volume of $9 \mu \mathrm{L}$ containing $20 \mathrm{mM}$ sodium citrate ( $\mathrm{pH} 5.0$ ), $1 \mathrm{mM}$ EDTA, and $7 \mathrm{M}$ urea, and were then successively incubated at $90^{\circ} \mathrm{C}$ for $1 \mathrm{~min}$ and at room temperature for $1 \mathrm{~min}$. Then, $1 \mu \mathrm{L}$ of a specific RNase ( $0.5 \mathrm{U}$ of RNase T1, $0.25 \mathrm{U}$ of either RNase U or RNase TA, or $1 \mathrm{pg} / \mu \mathrm{l}$ of RNase A) was added, the mixtures incubated at room temperature for $5 \mathrm{~min}$ and the reactions then quenched by the addition of $10 \mu \mathrm{L} 50 \%$ formamide and $10 \mathrm{mM}$ EDTA solution. In the case of alkaline hydrolysis the transcripts were dissolved in a mixture of $9 \mu \mathrm{L}$ of water and $1 \mu \mathrm{L}$ of $2 \mathrm{~N}$ $\mathrm{NaOH}$, and were then incubated at room temperature for $1 \mathrm{~min}$. These latter reactions were quenched by the addition of $10 \mu \mathrm{L}$ of $1 \mathrm{M}$ Tris- $\mathrm{HCl}$ ( $\mathrm{pH} 7.5$ ), the RNA was then ethanol precipitated in the presence of glycogen and subsequently dissolved in $10 \mu \mathrm{L}$ $50 \%$ formamide and $10 \mathrm{mM}$ EDTA solution. All reactions were fractionated on either $5 \%$ or $8 \%$ denaturing PAGE gels, and then subsequently visualized by exposure of the gels to phosphor imaging screens.

\section{Electrophoretic mobility shift assays}

DNA oligonucleotides complementary to different regions of PLMVd transcripts were 5 '-end labeled as described above. These oligonucleotides were purified on denaturing 20\% PAGE gels, excised, eluted overnight in elution buffer, and then ethanol precipitated in the presence of glycogen. Transcripts were heated at $65^{\circ} \mathrm{C}-70^{\circ} \mathrm{C}$ and then slow cooled in order to ensure proper folding. PLMVd transcripts $(30 \mu \mathrm{M})$ and oligonucleotides (500 $\mathrm{cpm}$ ) were mixed together in either a solution containing $50 \mathrm{mM}$ Tris- $\mathrm{HCl}(\mathrm{pH} 7.5)$ and $10 \mathrm{mM} \mathrm{MgCl}_{2}$, or in one containing $10 \mathrm{mM}$ Tris- $\mathrm{HCl}(\mathrm{pH} \mathrm{7.5})$ and $1 \mathrm{mM} \mathrm{MgCl}_{2}$, in a final volume of $10 \mu \mathrm{L}$. After an incubation of $10 \mathrm{~min}$ at $25^{\circ} \mathrm{C}, 10 \mu \mathrm{L}$ of loading buffer were added prior to electrophoresing overnight at $4^{\circ} \mathrm{C}$ through native $20 \%$ polyacrylamide gels (29:1 acrylamide:bisacrylamide ratio) in $45 \mathrm{mM}$ Tris-borate $(\mathrm{pH} 7.5)$ and $150 \mathrm{mM} \mathrm{NaCl}$ buffer. The gels were exposed to phosphor screens, which were then revealed using a Storm scanner (Molecular Dynamics). Analyses were performed using both the Image Quant and Prism softwares.

\section{RNase $\mathbf{H}$ digestion}

PLMVd transcripts $(<1 \mathrm{nM}, \sim 10,000 \mathrm{cpm})$ were heat denatured and slow cooled prior to being added to a solution containing $20 \mathrm{mM}$ HEPES-KOH (pH 8.0), $50 \mathrm{mM} \mathrm{KCl}, 4 \mathrm{mM} \mathrm{MgCl}_{2}, 1 \mathrm{mM}$ DTT, and $1 \mu \mathrm{M}$ of a specific oligonucleotide in a final volume of $10 \mu \mathrm{L}$. One unit of RNase $\mathrm{H}$ was added and the mixtures incubated at $37^{\circ} \mathrm{C}$ for $10 \mathrm{~min}$, at which point one volume of stop buffer was added. The T1 RNA ladders were prepared as described 
in the enzymatic probing of PLMVd strands section. After incubation, the reactions were heated at $65^{\circ} \mathrm{C}$ for $5 \mathrm{~min}$ before being fractionated on denaturing $8 \%$ PAGE gels. The gels were exposed to phosphor screens, which were then revealed using a Storm scanner (Molecular Dynamics).

\section{SUPPLEMENTAL MATERIAL}

Supplemental material can be found at http://www.rnajournal.org.

\section{ACKNOWLEDGMENTS}

This work was supported by a grant from the Natural Sciences and Engineering Research Council (NSERC Canada, grant number 155219-07) to J.-P.P. The RNA group is supported by grants from the Universite de Sherbrooke and the Canadian Institutes of Health Research (CIHR, grant number PRG-80169). A.D. was the recipient of predoctoral fellowships from both NSERC and Fonds québécois de la recherche sur la nature et les technologies (FQRNT). J.-P.P. holds the Canada Research Chair in Genomics and Catalytic RNA. M.B. and J.-P.P. are members of the Centre de Recherche Clinique Étienne-Lebel.

Received July 16, 2009; accepted November 26, 2009.

\section{REFERENCES}

Ambros S, Hernandez C, Desvignes JC, Flores R. 1998. Genomic structure of three phenotypically different isolates of peach latent mosaic viroid: Implications of the existence of constraints limiting the heterogeneity of viroid quasispecies. J Virol 72: 7397-7406.

Amitsur M, Levitz R, Kaufmann G. 1987. Bacteriophage T4 anticodon nuclease, polynucleotide kinase and RNA ligase reprocess the host lysine tRNA. EMBO J 6: 2499-2503.

Baumstark T, Riesner D. 1995. Only one of four possible secondary structures of the central conserved region of potato spindle tuber viroid is a substrate for processing in a potato nuclear extract. Nucleic Acids Res 23: 4246-4254.

Baumstark T, Schroeder AR, Riesner D. 1997. Viroid processing: Switch from cleavage to ligation is driven by a change from a tetraloop to a loop E conformation. EMBO J 16: 599-610.

Beaudry D, Bussière F, Lareau F, Lessard C, Perreault JP. 1995. The RNA of both polarities of the peach latent mosaic viroid selfcleaves in vitro solely by single hammerhead structures. Nucleic Acids Res 23: 745-752.

Bussière F, Lafontaine D, Perreault JP. 1996. Compilation and analysis of viroid and viroid-like RNA sequences. Nucleic Acids Res 24: 1793-1798.

Bussière F, Ouellet J, Côté F, Lévesque D, Perreault JP. 2000. Mapping in solution shows the peach latent mosaic viroid to possess a new pseudoknot in a complex, branched secondary structure. J Virol 74: 2647-2654.

Chaffai M, Serra P, Gandia M, Hernandez C, Duran-Vila N. 2007. Molecular characterization of CEVd strains that induce different phenotypes in Gynura aurantiaca: Structure-pathogenicity relationships. Arch Virol 152: 1283-1294.

Delgado S, Martinez de Alba AE, Hernandez C, Flores R. 2005. A short double-stranded RNA motif of Peach latent mosaic viroid contains the initiation and the self-cleavage sites of both polarity strands. J Virol 79: 12934-12943.

Diener TO. 2003. Discovering viroids-a personal perspective. Natl Rev 1: 75-80.
Dingley AJ, Steger G, Esters B, Riesner D, Grzesiek S. 2003. Structural characterization of the 69 nucleotide potato spindle tuber viroid left-terminal domain by NMR and thermodynamic analysis. $J \mathrm{Mol}$ Biol 334: 751-767.

Dubé A, Bisaillon M, Perreault JP. 2009. Identification of proteins from Prunus persica interacting with peach latent mosaic virus. J Virol 83: 12057-12067.

Gago S, De la Pena M, Flores R. 2005. A kissing-loop interaction in a hammerhead viroid RNA critical for its in vitro folding and in vivo viability. RNA 11: 1073-1083.

Gast FU, Spieker RL. 1996. Viroids proper can be distinguished from hammerhead viroids and satellite RNAs through their dinucleotide composition. Arch Virol 141: 1775-1783.

Gross HJ, Domdey H, Lossow C, Jank P, Raba M, Alberty H, Saenger HL. 1978. Nucleotide sequence and secondary structure of potato spindle tuber viroid. Nature 273: 203-208.

Lafontaine D, Beaudry D, Marquis P, Perreault JP. 1995. Intra- and intermolecular nonenzymatic ligations occur within transcripts derived from the peach latent mosaic viroid. Virology 212: 705709.

Navarro B, Flores R. 1997. Chrysanthemum chlorotic mottle viroid: Unusual structural properties of a subgroup of self-cleaving viroids with hammerhead ribozymes. Proc Natl Acad Sci 94: 11262-11267.

Owens RA. 2007. Potato spindle tuber viroid: The simplicity paradox resolved? Mol Plant Pathol 8: 549-560.

Pelchat M, Deschenes P, Perreault JP. 2000. The database of the smallest known auto-replicable RNA species: Viroids and viroidlike RNAs. Nucleic Acids Res 28: 179-180.

Qi Y, Ding B. 2003. Inhibition of cell growth and shoot development by a specific nucleotide sequence in a noncoding viroid RNA. Plant Cell 15: 1360-1374.

Qu F, Heinrich C, Loss P, Steger G, Tien P, Riesner D. 1993. Multiple pathways of reversion in viroids for conservation of structural elements. EMBO J 12: 2129-2139.

Repsilber D, Wiese S, Rachen M, Schroeder AW, Riesner D, Steger G. 1999. Formation of metastable RNA structures by sequential folding during transcription: Time-resolved structural analysis of potato spindle tuber viroid (-)-stranded RNA by temperaturegradient gel electrophoresis. RNA 5: 574-584.

Riesner D, Steger G. 2005. Temperature-gradient gel electrophoresis of RNA. Handbook of RNA biochemistry, pp. 398-414. Wiley-VCH, Weinheim, Germany.

Rosenbaum V, Riesner D. 1987. Temperature-gradient gel electrophoresis. Thermodynamic analysis of nucleic acids and proteins in purified form and in cellular extracts. Biophys Chem 26: 235-246.

Schmitz M, Steger G. 2007. Potato spindle tuber viroid (PSTVd). Plant Viruses 1: 106-115.

Schnoelzer M, Haas B, Raam K, Hofmann H, Saenger HL. 1985. Correlation between structure and pathogenicity of potato spindle tuber viroid (PSTV). EMBO J 4: 2181-2190.

Schrader O, Baumstark T, Riesner D. 2003. A mini-RNA containing the tetraloop, wobble-pair and loop E motifs of the central conserved region of potato spindle tuber viroid is processed into a minicircle. Nucleic Acids Res 31: 988-998.

Schroeder AR, Riesner D. 2002. Detection and analysis of hairpin II, an essential metastable structural element in viroid replication intermediates. Nucleic Acids Res 30: 3349-3359.

Schumacher J, Meyer N, Riesner D, Weidemann HL. 1986. Diagnostic procedure for the detection o viroids and viruses with circular RNA by return-gel electrophoresis. J Phytopathol 115: 332-343.

Zhong X, Archual AJ, Amin AA, Ding B. 2008. A genomic map of viroid RNA motifs critical for replication and systemic trafficking. Plant Cell 20: $35-47$.

Zhong X, Leontis N, Qian S, Itaya A, Qi Y, Boris-Lawrie K, Ding B. 2006. Tertiary structural and functional analyses of a viroid RNA motif by isostericity matrix and mutagenesis reveal its essential role in replication. J Virol 80: 8566-8581. 

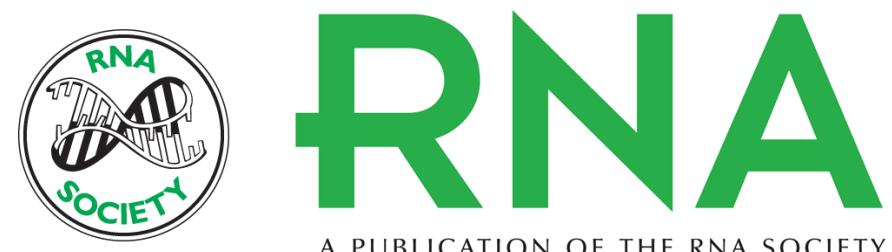

A PUBLICATION OF THE RNA SOCIETY

\section{The RNA strands of the plus and minus polarities of peach latent mosaic viroid fold into different structures}

Audrey Dubé, Tilman Baumstark, Martin Bisaillon, et al.

RNA 2010 16: 463-473 originally published online January 20, 2010

Access the most recent version at doi:10.1261/rna.1826710

\section{Supplemental http://rnajournal.cshlp.org/content/suppl/2010/01/08/rna.1826710.DC1 \\ Material}

References This article cites 30 articles, 11 of which can be accessed free at: http://rnajournal.cshlp.org/content/16/3/463.full.html\#ref-list-1

\section{License}

Email Alerting Receive free email alerts when new articles cite this article - sign up in the box at the Service top right corner of the article or click here.

\section{|||||||| Providing Precise Solutions for your research.}

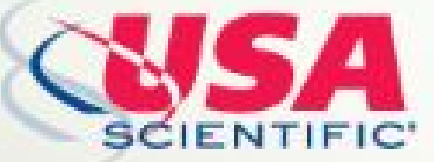

To subscribe to $R N A$ go to:

http://rnajournal.cshlp.org/subscriptions 